Article

\title{
Tree Lab: Portable Genomics for Early Detection of Plant Viruses and Pests in Sub-Saharan Africa
}

\author{
Laura M. Boykin ${ }^{1, *(\mathbb{C}, \text { Peter Sseruwagi }}{ }^{2}$, Titus Alicai ${ }^{3}$, Elijah Ateka ${ }^{4}$, \\ Ibrahim Umar Mohammed ${ }^{5}$, Jo-Ann L. Stanton ${ }^{6}$, Charles Kayuki ${ }^{2}$, Deogratius Mark ${ }^{2}$, \\ Tarcisius Fute ${ }^{2}$, Joel Erasto ${ }^{2}$, Hilda Bachwenkizi ${ }^{2}$, Brenda Muga ${ }^{4}$, Naomi Mumo 4 (iD, \\ Jenniffer Mwangi ${ }^{4}$ (D), Phillip Abidrabo ${ }^{3}$, Geoffrey Okao-Okuja ${ }^{3}$, Geresemu Omuut ${ }^{3}$, \\ Jacinta Akol ${ }^{3}$, Hellen B. Apio ${ }^{3}$, Francis Osingada ${ }^{3}$, Monica A. Kehoe ${ }^{7}$, David Eccles ${ }^{8}$ (D), \\ Anders Savill ${ }^{1}$, Stephen Lamb ${ }^{1}$, Tonny Kinene ${ }^{1}$, Christopher B. Rawle ${ }^{6}$ (D), \\ Abishek Muralidhar ${ }^{9}$, Kirsty Mayall ${ }^{9}$ (D), Fred Tairo ${ }^{2}$ and Joseph Ndunguru ${ }^{2}$ \\ 1 School of Molecular Sciences and Australian Research Council Centre of Excellence in Plant Energy Biology, \\ University of Western Australia, Crawley, Perth, WA 6009, Australia \\ 2 Mikocheni Agricultural Research Institute (MARI), Dar es Salaam P.O. Box 6226, Tanzania \\ 3 National Crops Resources Research Institute (NaCRRI), Kampala P.O. Box 7084, Uganda \\ 4 Jomo Kenyatta University of Agriculture and Technology (JKUAT), Nairobi P.O. Box 62000-00200, Kenya \\ 5 Department of Crop Science, Faculty of Agriculture, Kebbi State University of Science and Technology, \\ Aliero P.O. Box 1144, Nigeria \\ 6 Department of Anatomy, University of Otago, P.O. Box 56, Dunedin 9054, New Zealand \\ 7 Department of Primary Industries and Regional Development Diagnostic Laboratory Services, \\ Plant Pathology, South Perth, WA 6151, Australia \\ 8 Malaghan Institute of Medical Research, P.O. Box 7060, Newtown, Wellington 6242, New Zealand \\ 9 MicroGEM Ltd., 9 Melody Ln, Ruakura, Hamilton 3216, New Zealand \\ * Correspondence: laura.boykin@uwa.edu.au; Tel.: +61-8-6488-4488
}

Received: 26 July 2019; Accepted: 19 August 2019; Published: 21 August 2019

Abstract: In this case study we successfully teamed the PDQeX DNA purification technology developed by MicroGEM, New Zealand, with the MinION and MinIT mobile sequencing devices developed by Oxford Nanopore Technologies to produce an effective point-of-need field diagnostic system. The PDQeX extracts DNA using a cocktail of thermophilic proteinases and cell wall-degrading enzymes, thermo-responsive extractor cartridges and a temperature control unit. This closed system delivers purified DNA with no cross-contamination. The MinIT is a newly released data processing unit that converts MinION raw signal output into nucleotide base called data locally in real-time, removing the need for high-specification computers and large file transfers from the field. All three devices are battery powered with an exceptionally small footprint that facilitates transport and setup. To evaluate and validate capability of the system for unbiased pathogen identification by real-time sequencing in a farmer's field setting, we analysed samples collected from cassava plants grown by subsistence farmers in three sub-Sahara African countries (Tanzania, Uganda and Kenya). A range of viral pathogens, all with similar symptoms, greatly reduce yield or destroy cassava crops. Eight hundred (800) million people worldwide depend on cassava for food and yearly income, and viral diseases are a significant constraint to its production. Early pathogen detection at a molecular level has great potential to rescue crops within a single growing season by providing results that inform decisions on disease management, use of appropriate virus-resistant or replacement planting. This case study presented conditions of working in-field with limited or no access to mains power, laboratory infrastructure, Internet connectivity and highly variable ambient temperature. An additional challenge is that, generally, plant material contains inhibitors of downstream molecular processes making effective DNA purification critical. We successfully undertook real-time on-farm genome sequencing of samples collected from cassava plants on three farms, one in each country. Cassava mosaic begomoviruses were detected by sequencing leaf, stem, tuber and insect samples. 
The entire process, from arrival on farm to diagnosis, including sample collection, processing and provisional sequencing results was complete in under $3 \mathrm{~h}$. The need for accurate, rapid and on-site diagnosis grows as globalized human activity accelerates. This technical breakthrough has applications that are relevant to human and animal health, environmental management and conservation.

Keywords: cassava; cassava mosaic begomovirus; cassava mosaic disease; Bemisia tabaci; whitefly; MinION; MinIT; PDQeX; Tanzania; Uganda; Kenya

\section{Introduction}

Crop losses due to viral diseases and pests are major constraints on food security and income for millions of households in sub-Saharan Africa (SSA). Such losses can be reduced if plant diseases and pests are correctly diagnosed and identified early. To date, researchers have utilized conventional methods for definitive identification of plant viruses and their vectors in SSA including PCR, qPCR (quantitative PCR), next generation and Sanger sequencing, but these require laboratory infrastructure, are costly and time consuming and can delay time-sensitive corrective actions that could be taken. Direct rapid DNA/RNA sequencing of infected material on-the-spot or near sample collection sites turns this conventional paradigm on its head by taking the laboratory closer to farmers' fields. This reduces overall costs and gives crop protection officers and farmers in rural communities' information that is critical for sustainable crop production and management of pests and diseases, ensuring food and income security for millions of Africans. Currently, provision of data on viruses which are essential for developing virus resistant varieties, sharing virus-indexed germplasm between regions and deployment of virus-free certified planting materials is hampered by the long time taken to receive results generated using the aforementioned conventional diagnostic methods. Our innovation will simplify information flow and fast-track the deployments of virus resistant or tolerant cassava varieties directly to the farmer's field. The emergence of new tools for real-time diagnostics, such as the Oxford Nanopore MinION, has proved useful for the early detection of Ebola [1] and Zika viruses [2,3]. MinION consensus sequence accuracy of $99 \%$ is sufficient to identify pathogen and strain type [4]. However, it can take months before results generated using other high-throughput sequencing approaches (e.g., Illumina, PacBio) are available, particularly when local scientists are reliant on third-party service providers, who are often located in other countries. The delay in detecting or identifying viruses impedes quick in situ decision-making necessary for early action, crop protection advice and disease management strategies by farmers. This ultimately compounds the magnitude of crop losses and food shortages suffered by farmers. We have decreased the time to precisely detect and identify pathogens, vectors or pests, and increased resolution and reliability of results by utilizing the power of low-cost portable DNA extraction, sequencing and data analysis devices, coupled with our innovative data analysis pipelines. This real-time diagnosis in the field or located in regional laboratories quickly provides high quality and reliable diagnostics data to help farmers, seed certification agencies, scientists, crop protection and extension officers make timely and informed decisions. The immediate data accessibility makes possible dissemination of results downstream to extension officers and farmers for early disease control action via Information and Communication Technologies (ICT) applications. The application of cutting-edge sequencing technology, genomics and bioinformatics for pest and disease control has great potential to improve food security and agricultural development at large.

We propose using this technology to rapidly diagnose plant viruses and pests affecting smallholder farmers' crops in SSA. Our case study has identified cassava DNA viruses on the farm allowing farmers, researchers and development actors to take early, corrective action based on the rapid diagnosis of plants. This proof-of-concept shows that portable DNA sequencer technology has great potential to reduce the risk of community crop failure. We have previously conducted pilot projects in Tanzania, Uganda and Kenya testing symptomatic and asymptomatic cassava plants and 
have already shown that sample collection to diagnosis and results delivered to the farmer or crop protection officer can be completed within $48 \mathrm{~h} \mathrm{[5].} \mathrm{This} \mathrm{technology} \mathrm{will} \mathrm{put} \mathrm{the} \mathrm{power} \mathrm{of} \mathrm{genome}$ sequencing directly in the hands of agriculturalists and, in the work presented here, for the first time has enabled pest and disease diagnosis within one day on-the-spot. We have documented our experiences at https:/cassavavirusactionproject.com. This has significant implications for new pest and disease outbreaks, monitoring of existing disease outbreaks and biosecurity monitoring at borders between countries.

\section{Materials and Methods}

\subsection{Tree Lab Locations}

All essential equipment that were used in the Tree Labs in Kenya, Uganda and Tanzania are listed in Table 1 and shown in Figure 1. Three small-scale family farms growing cassava were selected, one in each of the following counties: Tanzania, Uganda and Kenya. In Tanzania (Kisamwene,

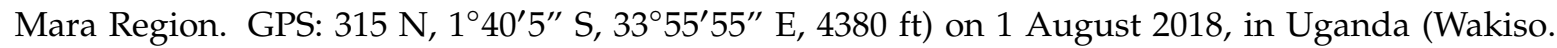
GPS: 255 W $0^{\circ} 30^{\prime} 29^{\prime \prime} \mathrm{N}, 32^{\circ} 37^{\prime} 19^{\prime \prime} \mathrm{E}, 3730 \mathrm{ft}$ ) on 8 August 2018, and in Kenya (Kiambu. GPS: $87 \mathrm{E}$ $1^{\circ} 5^{\prime} 33^{\prime \prime}$ S, 37 $19^{\prime} 33^{\prime \prime} \mathrm{E}, 4570 \mathrm{ft}$ ) on 14 August 2018. A video of the Tanzanian Tree Lab is found here: https://vimeo.com/329068227.

Table 1. Essential equipment for Tree Lab in Tanzania, Kenya and Uganda.

\begin{tabular}{|c|}
\hline 1. Sample Collection Plants \\
\hline $\begin{array}{l}\text { Envelopes } \\
4 \text { fine Sharpie markers } \\
\text { Notebook } \\
\text { Scissors } \\
\text { Canvas sling bag to carry sample collection equipment } \\
\text { Gloves }\end{array}$ \\
\hline 2. Sample collection whiteflies \\
\hline $\begin{array}{l}\text { Pooter } \\
70 \% \text { ETOH } \\
\text { Eppendorf tubes } \\
\text { Transfer pipette to get the whitefly from the pooter to the Eppendorf tube }\end{array}$ \\
\hline 3. Lab under a tree/disease diagnostic camp \\
\hline $\begin{array}{l}\text { Blanket/floor covering } \\
\text { Hard flat surface- raised }\end{array}$ \\
\hline 4. Sample homogenization \\
\hline $\begin{array}{l}1 \text { microfuge tube/sample } \\
1 \mathrm{~mL} \text { tips with fine tip sealed to form pestle for crushing } \\
\text { Tip disposal/waste bags } \\
1.5 \mathrm{~mL} \text { racks for holding } \\
\text { p10, p1000, p200 pipette (one set of pipettes)- tips for all } 1 \text { box }\end{array}$ \\
\hline 5. DNA extraction \\
\hline $\begin{array}{l}2 \text { MicroGEM kits (transported at ambient temperature) } \\
\quad \text { PDQeX phytoGEM kit for plants } \\
\text { PDQex Universal kit for insects } \\
\text { Reagents lyophilized into } 200 \mu \mathrm{L} \text { tubes } \\
\text { Rack for } 200 \mu \mathrm{L} \text { microcentrifuge tubes } \\
\text { Nuclease free water } \\
\text { PDQeX thermal device } \\
\text { PDQeX Extraction tubes } \\
\text { Mobile phone being used as a remote controller- MicroGEM PDQeX app } \\
\text { Battery } 212 \mathrm{~V} \text { batteries put together }\end{array}$ \\
\hline
\end{tabular}


Table 1. Cont.

\begin{tabular}{l}
\hline 6. Save the DNA for later quantification using fluorometry (e.g, Qubit). \\
\hline 7. Library prep \\
\hline Printed library prep protocol or iPad \\
Library kit \\
$200 \mu$ L tubes \\
$1.5 \mathrm{~mL}$ tubes \\
Ampure beads \\
Magnetic rack \\
Tube stands for the $0.2 \mathrm{~mL}$ \\
Tris and sodium chloride \\
Nuclease free water \\
Styrofoam cooler and cold packs \\
Flow cell \\
MinIT \\
Laptop Power bank-one with a pin plug not just USB output \\
Laptop- with MinKNOW installed
\end{tabular}

\subsection{Sample Selection}

All samples collected are documented in Table 2. For each sequencing run we barcoded 11 DNA extractions from cassava leaves, stems and whiteflies (Bemisia tabaci) that were found feeding on cassava mosaic disease symptomatic cassava leaves.

\subsection{DNA Extraction}

The PDQeX DNA extraction system from MicroGEM Ltd. (Hamilton, New Zealand) [6] was used to prepare DNA from samples. Briefly, a Harris punch was used to collect four discs, $2 \mathrm{~mm}$ in diameter, from each leaf, stem or root sample. Homogenization was performed by hand in $100 \mu \mathrm{L}$ of $1 \times$ GREEN+ buffer using a Dounce homogenizer made from sealing the end of a $1 \mathrm{~mL}$ pipette tip and a $1.5 \mathrm{~mL}$ microfuge tube. Ninety microlitres of each homogenate and $10 \mu \mathrm{L}$ of Enhancer (MicroGEM Ltd.) was added to a $200 \mu \mathrm{L}$ tube containing a lyophilized $1 \times$ mix of the enzyme cocktail [7]; (phytoGEM kit, MicroGEM Ltd.). The reaction was re-suspended by gently flicking the $200 \mu \mathrm{L}$ tube until all reagents were well mixed. All of the reaction mix was transferred to a PDQeX extraction cartridge [6] which was placed into the PDQeX1600 thermal incubation unit. PDQeX extraction was performed by a series of heating steps. First, incubation at $52{ }^{\circ} \mathrm{C}$ for five minutes was conducted to promote cell lysis by activating cell wall degrading enzymes. Second, incubation at $75^{\circ} \mathrm{C}$ for five minutes was conducted to activate thermophilic proteinases to degrade sample proteins and enzymes from the previous step. Finally, heating to $95^{\circ} \mathrm{C}$ for 2 min was conducted to shrink the thermal responsive inner layer of the PDQeX extraction cartridge forcing the digested sample through a burst valve and a clean-up column into a collection tube [6].

DNA was also extracted from whitefly. A single insect was fished from a pool of whiteflies in ethanol collected from leaves using a Pooter. The whitefly was transferred by pipette, taking as little ethanol as possible, to $98 \mu \mathrm{L}$ of $1 \times$ BLUE buffer (MicroGEM Ltd.) and pipetted up and down several times. The whole mix was added to $1 \times$ lyophilized enzyme cocktail in a $200 \mu \mathrm{L}$ tube (prepGEM, MicroGEM Ltd.). Reagents were re-suspended by gentle flicking and the contents transferred to a PDQeX extraction cartridge. The cartridge was placed in the PDQeX1600 thermal unit and heated as follows: $35^{\circ} \mathrm{C}$ for five minutes; $52{ }^{\circ} \mathrm{C}$ for five minutes; $75{ }^{\circ} \mathrm{C}$ for five minutes; $95^{\circ} \mathrm{C}$ for $2 \mathrm{~min}$. DNA extraction took approximately $20 \mathrm{~min}$ in total and $7.5 \mu \mathrm{L}$ of the collected elute was used directly for Rapid DNA library construction for MinION Sequencing (Oxford Nanopore Technologies, Oxford, United Kingdom). The PDQeX1600 thermal unit was powered by a 12-volt Lithium Polymer battery. The PDQeX1600 was operated using a purpose-made App from a smart phone that permitted run initiation, temperature profile selection and editing, and monitoring of run progress. 


\subsection{Library Preparation and Sequencing}

We utilized the Rapid Barcoding kit SQK-RBK004 with 9.4.1 flow cells (Oxford Nanopore Technologies). The SQK-RBK004 protocols were performed as described by the manufacturer (RBK_9054_v2_revB_23Jan2018). We completed the optional clean-up steps using AMPure XP beads (Beckman Coulter, Brea, California, United States of America). The $30{ }^{\circ} \mathrm{C}$ and $80{ }^{\circ} \mathrm{C}$ steps were performed using the PDQeX1600 thermal incubation unit. All libraries were loaded directly onto the MinION that was connected to a MinIT and live base calling was enabled. For each Tree Lab the MinION and the MinIT were plugged into a 20,000 mAh laptop powerbank (Comsol, Glendenning, New South Wales, Australia) set at $20 \mathrm{~V}$ (Figure 1). The key to using a power bank for this purpose is to make sure it not only has USB inputs but also has a DC port. It ran on average $4.5 \mathrm{~h}$ set on $16.5 \mathrm{~V}$. When the run stopped, we immediately plugged the devices into a second power bank and data generation continued.

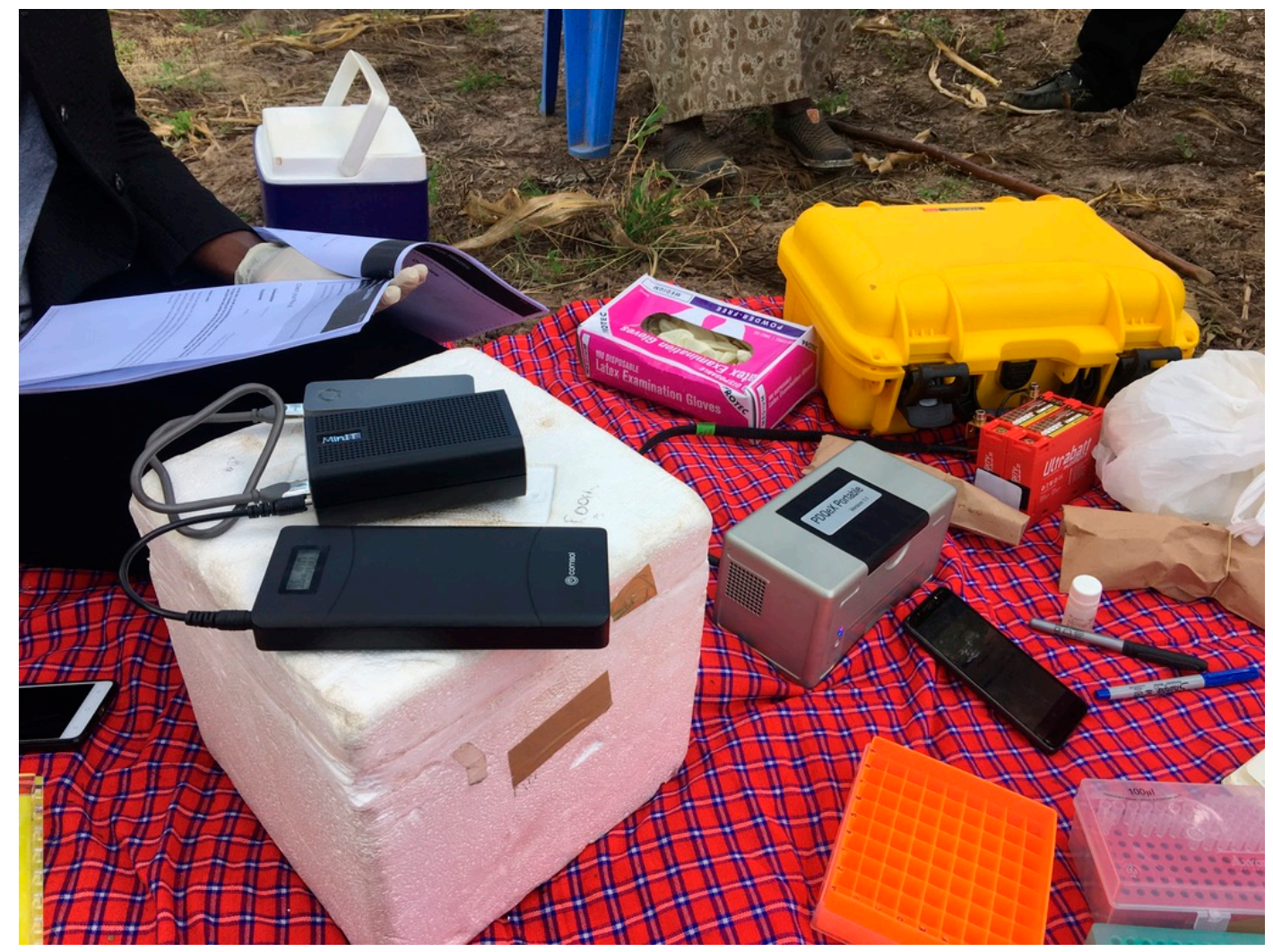

Figure 1. Tree Lab in Kenya. Essential equipment is listed in Table 1.

\subsection{Tree Lab Data Analyses}

As the data was being basecalled on the MinIT we made a test folder on the laptop called "treelab" and inside that folder we added a demultiplex folder, into which we then transferred the first two fastq files from the MinIT. Demultiplexing was run with Porechop [8], preinstalled on the laptop, using the following commands >porechop-i/Users/lboykin/Desktop/treelab-b/Users/lboykin/Desktop/treelab/demultiplex. A cassava mosaic disease (CMD) reference data set was pre-curated and configured to work as a local database within Geneious v.11.1.2 [9] (https://figshare.com/articles/Nanopore_sequencing_of_cassava_from_ Tanzania_Uganda_and_Kenya/6667409). Twelve folders were created in Geneious, and the associated fastq files from the "demultiplex" folder were drag and dropped into the relevant folder created within Geneious. BLASTn [10] analysis was performed, ensuring the local CMD database was specified. 
The results from the search against the CMD database were visualized in situ within Geneious and discussed with farmers and extension workers.

\subsection{Post Tree Lab Data Analyses}

Scripts from David Eccles' (Bioinformatics Scripts repository) [11] were used to carry out subsequent read quality control $(\mathrm{QC})$ and analysis. Sequenced read lengths were measured using fastx-length.pl, and these lengths were used to generate length-based QC plots using length_plot.r.

\subsection{Assembly}

To determine whether any barcoded read sets could be assembled, an initial assembly attempt was made on each subset using Canu v1.8, with a genome size of $400 \mathrm{M}$, ignoring any warning messages about coverage being too low. The large genome size ensured that no reads are discarded and suppressing the coverage warning ensured that Canu would attempt an assembly with all the available reads. Previous discussions with Canu developer Sergey Koren (pers. comm.) indicated that adjusting the target genome size had no other effect on the contig assemblies that Canu produces.

\subsection{Blast}

We confirmed these in-field results by performing a post diagnostic blast of reads on the Nimbus Cloud at the Pawsey Supercomputing Center with blast 2.2.31 against the full NCBI nucleotide database. For reference the specific database used was $\{\$ b l a s t c m d-d b$ nt $/$ nt-info\} Database: Nucleotide collection 49,266,009 sequences; 188,943,333,900 total bases Date: 8 August 2018, 12:38 PM. The data were processed into a blast archive using a blast script with the following parameters (Script attached) \{\$blastn-query “\$file"-db/mnt/nucdb/nt/nt-outfmt 11-culling_limit 10-out “out.\$file.asn"-num_threads 17) then converted into XML (for loading into Geneious) and HTML for viewing.

\subsection{Blastn Analysis-MEGAN}

Blastn results produced from the Nimbus cloud analysis pipeline were also visualized using MEGAN Community Edition version 6.12.6 [12] on the Zeus computing resource located at the Pawsey Supercomputing Center.

\subsection{Blastn Analysis-Kraken}

We used kraken2 [13] to classify demultiplexed reads using the Loman Lab "maxikraken2" database [14], on the Zeus computing resource located at the Pawsey Supercomputing Center.

\section{Results}

\subsection{Tree Lab}

Summary statistics for our three Tree Labs are shown in Table 2 and Figure 2. Table 2 summarizes DNA sequencing metrics from all three Tree Lab experiments. Each MinION run contained 11 barcoded libraries representing 11 individual samples. All DNA samples, except the African Cassava Mosaic Virus (ACMV) and East African Cassava Mosaic Virus (EACMV) controls (Tanzania), were extracted using the PDQeX system with 11 samples prepared in lab from exemplar material collected from scientific plots and 20 DNA samples extracted on farm. The controls were extracted using a CTAB approach described in [5]. A total of 1,442,599 sequences were produced across all the experiments. Of these, barcodes could only be resolved for 550,938 sequences using Porechop to demultiplex the samples. Mean sequence length across all sequencing runs ranged from 355-948 bp with the longest read being $276,793 \mathrm{bp}$.

Raw reads of cassava mosaic begomovirus (CMBs) sequences were detected in 21 samples, including the 2 controls, with the longest CMB read reaching $2808 \mathrm{bp}$, close to the full genome size. A total of 18 leaf samples were sequenced of which 15 were found to contain CMBs. Two of the 
five stem samples sequenced were found to contain CMBs whereas neither of the two root samples sequenced presented $\mathrm{CMB}$ sequences. Six single whiteflies were tested with two being positive for CMBs. All libraries, regardless of CMB content produced DNA reads indicating that sequencing was successful for all samples. CMBs were detected in plants with symptoms and there was a suggestion that the number of $\mathrm{CMB}$ sequences detected possibly correlated with symptom severity scores, but more data will be required to prove this. Interestingly, a known healthy plant taken from the scientific plot at JKUAT did not yield CMB sequences (Table 2).

Following assembly with Canu eight of the 21 samples gave complete assembled virus genomes. These eight assembled genomes have less than 10-fold coverage so further analyses were not pursued.

\subsection{Post Tree Lab Data Analyses}

We investigated whether there was any effect of sample type on read length. The cumulative density curves (Figure 2) show the proportion of sequenced bases with length greater than a particular length (with L10/L50/L90 highlighted). Additional length-based QC plots can be found in the supplemental information (Supplemental File 1).

\subsection{MEGAN Results}

The primary targets of this analysis were known cassava viruses, as well as the host, either cassava plant (Manihot esculenta) or the whitefly (Bemisia tabaci) and its endosymbionts. Results are summarized in Table 3 and, in general, the desired result of virus (EACMV or ACMV) and host DNA were recovered from all symptomatic samples.

\subsection{Kraken2 Results}

The analysis using Kraken 2 had an approximately $50 \%$ classification success rate (interquartile range- IQR $45-52 \%$ unclassified reads). This database is for human + microbial and viral sequence, so any eukaryote reads (e.g., from cassava or whitefly) would probably be unclassified by Kraken2 or assigned to the human taxa. The sample with the highest classification success was the ACMV positive control from Tanzania (mr_BC11, 5.6\% unclassified), and the lowest classification success was the leaf tissue sample \#2 from Kenya (mm_BC02, 61\% unclassified).

Results were aggregated into a table using Pavian (Supplemental File 2) to identify common elements of each sample. Begomovirus reads were detected in 15 samples, with very high proportions of Begomovirus $(8.6 \%)$ in the ACMV positive control from Tanzania (mr_BC11), and above-average proportions $(0.25 \%)$ in Kwatempale sample \#5 from Sarah's Farm in Uganda (ut_BC05). ACMV and EACMV were detected in 11 samples. 
Table 2. Summary statistics and locality information for the three Tree Labs in Tanzania, Kenya and Uganda. * indicates DNA extraction carried out using PDQeX in the laboratory before sequencing under the tree.

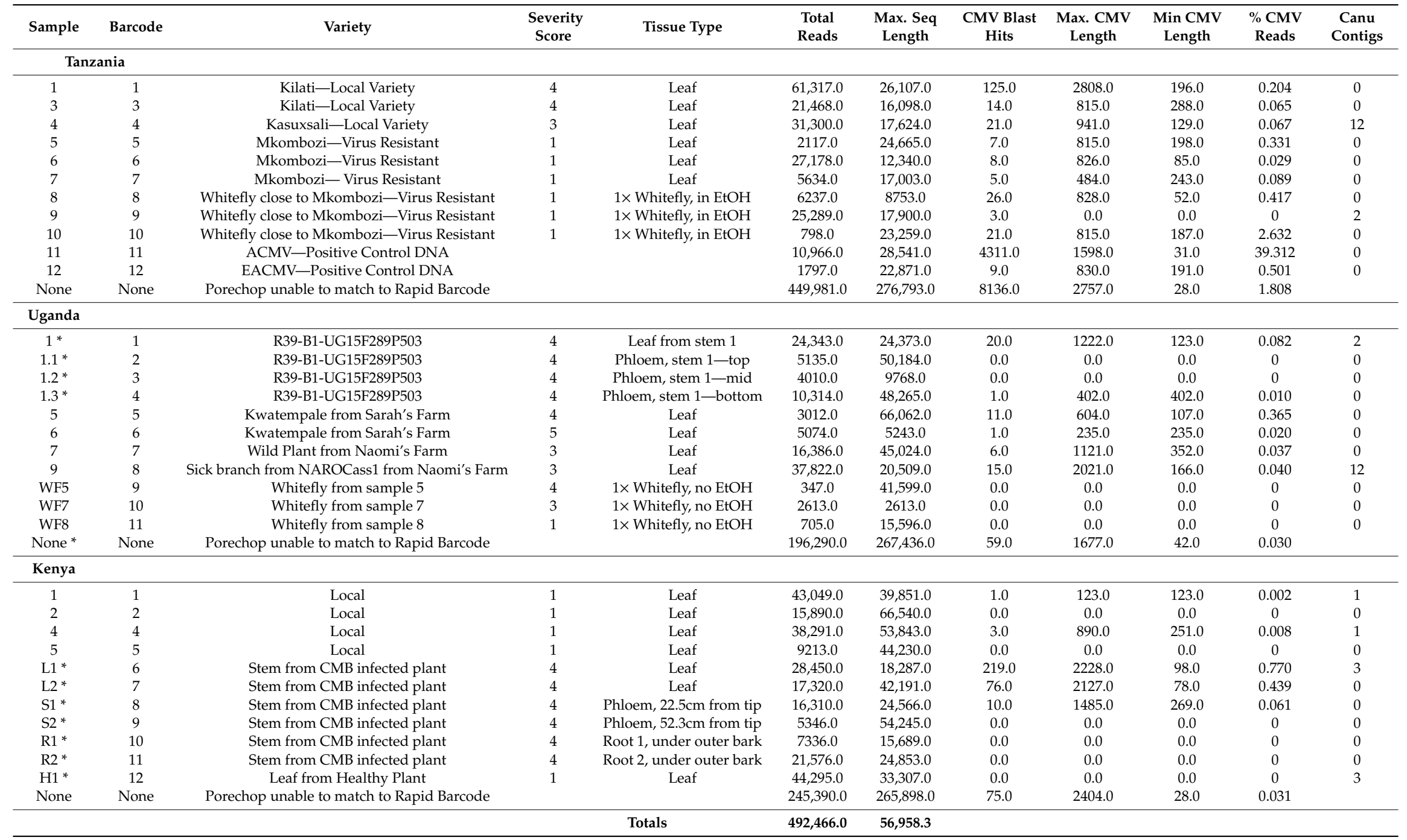


Table 3. Megan results of Blastn from Nimbus cloud.

\begin{tabular}{|c|c|c|c|c|c|c|c|c|c|c|c|}
\hline & $\begin{array}{c}\text { Total } \\
\text { Reads }\end{array}$ & $\begin{array}{l}\text { Reads } \\
\text { Classified }\end{array}$ & $\begin{array}{l}\text { Manihot } \\
\text { esculenta }\end{array}$ & EACMV & ACMV & TLCV & $\begin{array}{c}\text { Begomo-associated } \\
\text { DNA-III }\end{array}$ & $\begin{array}{c}\text { Bemisia } \\
\text { tabaci }\end{array}$ & $\begin{array}{l}\text { Bemisia } \\
\text { afer }\end{array}$ & $\begin{array}{l}\text { Candidatus Portiera } \\
\text { aleyrodidarum }\end{array}$ & Other \\
\hline \multicolumn{12}{|c|}{ Tanzania } \\
\hline 1 & 61,317 & 23,481 & 15,862 & 63 & - & 1 & & & & & \\
\hline 3 & 21,468 & 5407 & 3509 & 5 & & & & & & & \\
\hline 4 & 31,309 & 10,561 & 6462 & 2 & 5 & 1 & & & & & \\
\hline 5 & 2117 & 325 & 144 & & 2 & & & 3 & & 3 & \\
\hline 6 & 27,178 & 2127 & 76 & & 1 & & & 521 & 67 & 802 & \\
\hline 7 & 5634 & 1141 & 669 & & 1 & & & & & & \\
\hline 8 & 6237 & 1449 & 88 & & 5 & & & 912 & & 15 & \\
\hline 9 & 25,289 & 2303 & 126 & 2 & & & & 506 & 57 & 905 & \\
\hline 10 & 789 & 166 & 66 & & 3 & & & 11 & & 2 & \\
\hline 11 & 10,966 & 7843 & 69 & 3 & 616 & & & & & 2 & \\
\hline 12 & 1797 & 356 & 171 & & 2 & & & 13 & & 7 & \\
\hline \multicolumn{12}{|c|}{ Uganda } \\
\hline 1 & 18,853 & 5662 & 3073 & & 11 & & 1 & & & & \\
\hline 1.1 & 5135 & 876 & 402 & & & & & & & & \\
\hline 1.2 & 4010 & 1034 & 591 & & & & & & & & \\
\hline 1.3 & 10,314 & 1933 & 864 & & 1 & & & & & & \\
\hline 5 & 3012 & 556 & 255 & 1 & 7 & & & & & & \\
\hline 6 & 5074 & 758 & 39 & & 1 & & & & & & \\
\hline 7 & 16,386 & 4608 & 2666 & & 4 & & & & & & \\
\hline 9 & 37,822 & 12,768 & 7268 & 10 & & & & & & & \\
\hline WF5 & 347 & 51 & 20 & & & & & 10 & & & \\
\hline WF7 & 243 & 48 & 21 & & & & & & & & \\
\hline WF8 & 705 & 128 & 35 & & & & & 9 & & 1 & \\
\hline \multicolumn{12}{|c|}{ Kenya } \\
\hline 1 & 43,049 & 10,283 & 9947 & & 1 & 1 & 1 & & & & \\
\hline 2 & 15,890 & 2968 & 2854 & & & & & & & & \\
\hline 4 & 38,291 & 8648 & 8836 & 1 & 1 & & & & & & \\
\hline 5 & 8213 & 1959 & 1887 & & & & & & & & \\
\hline L1 & 28,450 & 9968 & 9580 & 45 & 81 & & & & & & SLCV (1) \\
\hline L2 & 17,320 & 4367 & 2050 & 29 & 4 & & & & & & \\
\hline S1 & 16,310 & 3718 & 1933 & 5 & & 1 & 1 & & & & \\
\hline R1 & 7336 & 2303 & 1016 & & & & & & & & \\
\hline R2 & 21,578 & 6810 & 2000 & & & & & & & & \\
\hline H1 & 44,295 & 15,537 & 8389 & & & & 1 & & & & \\
\hline
\end{tabular}


Tanzania

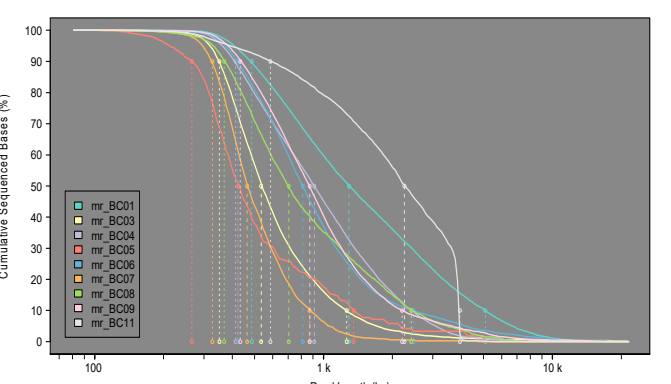

Figure 2. Cumulative density curves showing the proportion of sequenced bases with length greater than a particular length (with L10/L50/L90 highlighted).

Kenya
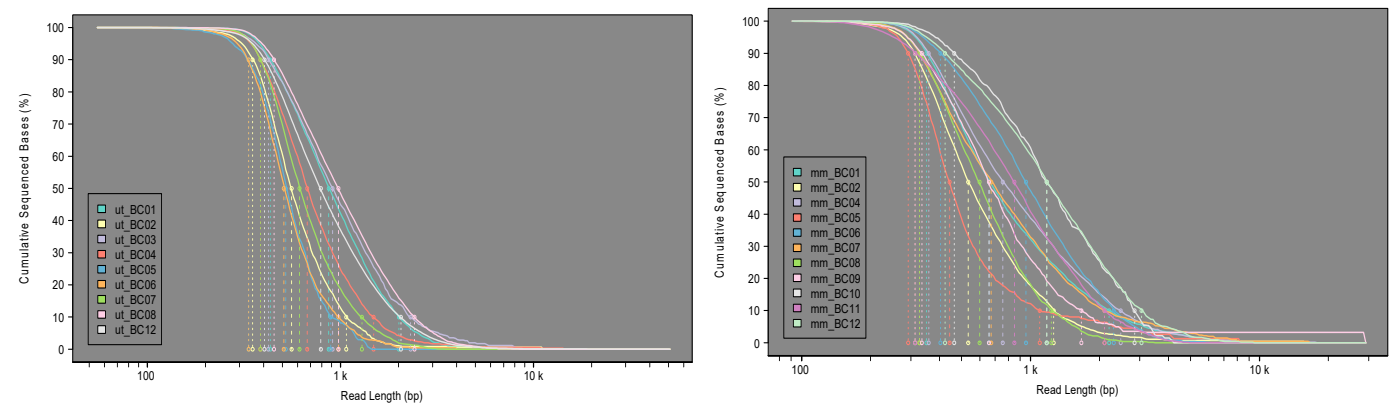

Uganda 


\section{Discussion}

This case study was designed to show the possibility to go from sample to diagnosis, in a regional setting, on farm in three hours versus the normal six months with conventional methods. The results of this research show that it is possible to use a range of battery powered devices to achieve DNA extraction, long read sequencing and analysis all under a tree on the farm while the farmers wait for results. Access to next-generation sequencing technology, or to services that offer access, has been a major barrier to their use in diagnostics for scientists, and particularly many agricultural scientists in SSA. The advent of the Oxford Nanopore Technologies MinION has brought this technology to their door in recent years, and with access to training through various institutions and especially the Oxford Nanopore Technologies run "Pore Safari" there are more and more users in the region. Previous studies that have used the technology for real time analysis of pathogen outbreaks, such as the Ebola and Zika studies [1-3] have still relied heavily on the transport of bulky laboratory equipment, or local acquisition of it to perform their work. Previous work by our team of scientists [5] showed that the turnaround time to result could be $48 \mathrm{~h}$, and now with the addition of the PDQeX and MinIT to the system we have been able to reduce the time to $3 \mathrm{~h}$ and perform the entire process in the field and under a tree. The cost for this run was 40 USD/sample for Oxford Nanopore reagents plus 3.70 USD/sample for DNA extraction materials from Microgem. This expense is much lower than the cost of losing a whole field of cassava. The estimated cost of 40 USD/sample is 10 times lower than the actual cost of testing the same sample for CMB in a public seed certifying institute in Tanzania (for example), where the approved cost per sample is 400 USD for cassava [15]. Furthermore, new technologies like the Flongle (the smaller flow cell from Oxford Nanopore at a cost of 90 USD) will soon reduce the cost per sample to 4 USD.

One of the major barriers to producing these outputs in the field has until now been the lack of a simple, quick and effective method to extract DNA from a sample without the need for laboratory equipment requiring mains power and space, items such as benchtop centrifuges, fridges, freezers and temperature- sensitive extraction kits which can be bulky and rely on traditional laboratory infrastructure. The use of the PDQeX in the system described in this case study was the real game changer: compact and able to operate from a battery, it made nucleic acid extraction possible.

This study also highlighted where the next gains for in-field sequencing are to be made, as improvements are required in rapid data analysis. The MinIT eliminated obstacles to base calling, by converting the raw reads into fast 5 and fastq reads in real-time. This moves the data analysis bottleneck in the pipeline to the Blast analysis. Blast is not a fast analysis, and so for now we must rely on a pre-curated database of known or expected pathogens and host genomes. This poses risks, in that new and emerging pathogens or vectors could be missed in the first instance, and not seen until subsequent data analysis when the scientist has returned to the lab or is within range of a good internet connection capable of uploading large amounts of data to the cloud. Even with this risk, there is no other choice for genomic sequencing rapidly in low resourced setting, the advantages of the methods outweigh the disadvantages. In our case, we can predict what sorts of genomes should be in our custom database, but for use in biosecurity and at borders between countries a better solution is required.

Read length distributions were generally quite similar for all samples. The Tanzania samples showed the greatest difference in read length distribution (L50 range 400-2000 bp). The ACMV Positive control from Tanzania (mr_BC11) showed a very pronounced spike of reads at around $4 \mathrm{~kb}$ (presumably near full-length ACMV sequence). Apart from that sample, there was no obvious association between read length distribution and tissue type or variety. The Uganda samples had a moderate read length distribution spread (L50 range 500-1000 bp), which split into two clusters of slightly shorter and longer reads ( $\mathrm{BC} 02, \mathrm{BC} 04, \mathrm{BC} 05, \mathrm{BC} 06, \mathrm{BC} 07 ; \mathrm{BC} 01, \mathrm{BC} 03, \mathrm{BC} 08, \mathrm{BC} 12)$. These clusters did not appear to have any relationship with tissue type or variety. The Kenya samples had a similarly moderate read length distribution spread (L50 range 500-1000 bp), with no obvious clustering or association of distribution with tissue type or variety. 
MinION Rapid libraries use transposase to fix sequencing adaptors to DNA fragments. The ratio of DNA to transposase complex for the MinION Rapid kit has been optimized for 400 ng DNA and at lower amounts DNA is susceptible to over fragmentation. This may account for DNA fragment length falling around $900 \mathrm{bp}$, however, the control DNA also gave similar read length characteristics. Though there was not enough data collected on farm for a thorough statistical analysis these results did show both yield and integrity of the DNA extracted using the PDQeX was of sufficient quality for diagnostic sequencing. We successfully retrieved enough data from each sample to establish whether the virus in the plant was EACMV or ACMV. Assembly with Canu suggested that, in this case, while there was enough data to assemble whole genomes, the average coverage meant the quality was not sufficient for downstream applications such as recombination detection and other evolutionary analyses. We anticipate that as the DNA extraction methods improve, and in-field library preparation becomes easier this will be possible. An alternative would be to investigate the use of a panel-like targeted amplicon approach or CRISPR/Cas9 enrichment but, again, this removes the likelihood of detecting unknowns in the samples, and could lead to samples giving negative results not being followed up, or the time to result being blown out to days or weeks if they need to return to a laboratory to complete a different type of library preparation. Compared with other in-field diagnostic tools, this system involving the MinION is unique in its ability to detect anything that might be present in the sample. Other in-field diagnostic tools, including serological based dipsticks, Loop-mediated isothermal amplification (LAMP)-PCR, in field qPCR and AI driven applications on smart phones all have one single thing in common-they require a prior knowledge of the suspected pathogen, coupled with the targeted design of antibodies, primers, or training for known positives to function effectively. The only decision required to run the MinION is whether to prepare a DNA or an RNA library.

\section{Executive Summary}

Can we go from sample to answer on the farm? Yes

DNA extraction to library prep to sequencing? Yes

Can we detect virus in leaves off the grid at the farm? Yes

Can we detect virus in whiteflies off the grid on the farm? Yes

Can we detect virus in stems off the grid on the farm? Yes

Do we get enough coverage of the viral genomes to generate polished genomes to track the evolution of the viruses real-time? No

Supplementary Materials: The following are available online at http://www.mdpi.com/2073-4425/10/9/632/ s1, Supplemental File 1: Additional length-based QC plots can be found in the supplemental information. Supplemental File 2: Kraken 2 results aggregated into a table using Pavian.

Author Contributions: Conceptualization, L.M.B., P.S., T.A., E.A., J.-A.L.S., C.K., M.A.K., F.T. and J.N.; Methodology, L.M.B., P.S., T.A., E.A., J.-A.L.S., C.K., B.M., P.A., G.O.-O., J.A., H.B.A., M.A.K., D.E., A.S. and J.N.; Software, J.-A.L.S., M.A.K., D.E., A.S., S.L., C.B.R., A.M., K.M.; Validation L.M.B., P.S., T.A., E.A., I.U.M., J.-A.L.S., C.K., D.M., T.F., J.E., H.B., B.M., N.M, J.M., P.A., G.O.-O., G.O., J.A., H.B.A., F.O., D.E., T.K., C.B.R., A.M. and J.N.; Formal Analysis, L.M.B.; J.-A.L.S., B.M., P.A., G.O.-O., J.A., H.B.A., M.A.K., D.E., A.S. and A.M.; Investigation, L.M.B., P.S., T.A., E.A., I.U.M., J.-A.L.S., C.K., D.M., T.F., J.E., H.B., B.M., N.M., J.M., P.A., G.O.-O., G.O., J.A., H.B.A., F.O., C.B.R., A.M., K.M. and J.N.; Resources, L.M.B., P.S., T.A., E.A., J.-A.L.S. and J.N.; Data Curation, L.M.B., C.K., B.M., P.A., G.O.-O., J.A., H.B.A., M.A.K., D.E. and A.S.; Writing-Original Draft Preparation, L.M.B., T.A., J.-A.L.S., M.A.K., D.E., S.L., F.T. and J.N.; Writing-Review \& Editing, L.M.B., P.S., T.A., E.A., I.U.M., J.-A.L.S., C.K., M.A.K., D.E., S.L., C.B.R.; Visualization, L.M.B., J.-A.L.S., M.A.K. and D.E.; Supervision, L.M.B., P.S., T.A., E.A., J.-A.L.S., C.K., M.A.K., F.T. and J.N.; Project Administration, L.M.B., P.S., T.A., E.A., J.-A.L.S., C.K., B.M., P.A., G.O.-O., M.A.K. and J.N.; Funding Acquisition, L.M.B., P.S., T.A., E.A., J.-A.L.S. and J.N.

Funding: This research received no external funding.

Acknowledgments: We are grateful to the farmers in Tanzania, Kenya and Uganda who allowed us access to their farms. This work is dedicated to them. We were honoured to have worked alongside scientist Jimmy Akono who passed earlier this year in Uganda. Special thank you to our drivers Honest Kway, Benson Ongori, and Jimmy Sebayiga. Filming was done by Andrew Court. PDQeX was developed under MBIE UOOX1507, New Zealand. Resources provided by Pawsey Supercomputing Centre with Funding from the Australia Government and the Government of Western Australia supported computation analysis. 
Conflicts of Interest: A. Muralidhar and K. Mayall are employees of MicroGEM. They provided protocol advice and custom lyophilised enzyme mixes for DNA extraction.

\section{References}

1. Quick, J.; Loman, N.J.; Duraffour, S.; Simpson, J.T.; Severi, E.; Cowley, L.; Ouédraogo, N.; Williams, V.; Amburgey, L.; Winona, E.; et al. Real-time, portable genome sequencing for Ebola surveillance. Nature 2016, 530, 228-232. [CrossRef] [PubMed]

2. Faria, N.R.; Sabino, E.C.; Nunes, M.R.; Alcantara, L.C.J.; Loman, N.J.; Pybus, O.G. Mobile real-time surveillance of Zika virus in Brazil. Genome Med. 2016, 8, 97. [CrossRef] [PubMed]

3. Quick, J.; Grubaugh, N.D.; Pullan, S.T.; Claro, I.M.; Smith, A.D.; Gangavarapu, K.; Burton, D.R.; Sabino, S.A.; Baylis, N.R.; Faria, M.; et al. Multiplex PCR method for MinION and Illumina sequencing of Zika and other virus genomes directly from clinical samples. Nat. Protoc. 2017, 12, 1261-1276. [CrossRef] [PubMed]

4. Calus, S.T.; Ijaz, U.Z.; Pinto, A.J. NanoAmpli-Seq: A workflow for amplicon sequencing for mixed microbial communities on the nanopore sequencing platform. Gigascience 2018, 7, giy140. [CrossRef] [PubMed]

5. Boykin, L.; Ghalab, A.; De Marchi, B.R.; Savill, A.; Wainaina, J.M.; Kinene, T.; Tairo, F.; Rodrigues, M.; Kehoe, J.; Ndunguru, F.; et al. Real time portable genome sequencing for global food security. F1000Research 2018, 7, 1101. [CrossRef]

6. Stanton, J.A.L.; Muralidhar, A.; Rand, C.J.; Saul, D.J. Rapid extraction of DNA suitable for NGS workflows from bacterial cultures using the PDQeX. BioTechniques 2019, 66, 208-213. [CrossRef] [PubMed]

7. Holmes, A.S.; Roman, M.G.; Hughes-Stamm, S. In-field collection and preservation of decomposing human tissues to facilitate rapid purification of STR typing. Forensic Sci. Int. Genet. 2018, 36, 124-129. [CrossRef] [PubMed]

8. Wick, R.R. Porechop. Available online: https://github.com/rrwick/Porechop (accessed on 13 July 2018).

9. Kearse, M.; Moir, R.; Wilson, A.; Stones-Havas, S.; Cheung, M.; Sturrock, S.; Buxton, S.; Cooper, A.; Markowitz, S.; Duran, C.; et al. Geneious Basic: An integrated and extendable desktop software platform for the organization and analysis of sequence data. Bioinformatics 2012, 28, 1647-1649. [CrossRef] [PubMed]

10. Altschul, S.F.; Gish, W.; Miller, W.; Myers, E.W.; Lipman, D.J. Basic local alignment search tool. J. Mol. Biol. 1990, 215, 403-410. [CrossRef]

11. Gringer/Bioinfscripts: Tree Lab/Global River Release. Available online: https://doi.org/10.5281/zenodo.596663 (accessed on 6 June 2019).

12. Huson, D.H.; Beier, S.; Flade, I.; Górska, A.; El-Hadidi, M.; Mitra, S.; Tappu, R.; Ruscheweyh, H.J.; Tappu, R. MEGAN community edition-interactive exploration and analysis of large-scale microbiome sequencing data. PLoS Comput. Biol. 2016, 12, e1004957. [CrossRef] [PubMed]

13. Wood, D.E.; Salzberg, S.L. Kraken: Ultrafast metagenomic sequence classification using exact alignments. Genome Biol. 2014, 15, R46. [CrossRef] [PubMed]

14. Maxikraken2_1903_140GB (March 2019, 140GB). Available online: https://lomanlab.github.io/ mockcommunity/mc_databases.html\#maxikraken2_1903_140gb-march-2019-140gb (accessed on 12 April 2019).

15. The Seed (Amendments) Regulations. Gazette of the United Republic of Tanzania No. 3, Vol. 98 5-13. 2017.

(C) 2019 by the authors. Licensee MDPI, Basel, Switzerland. This article is an open access article distributed under the terms and conditions of the Creative Commons Attribution (CC BY) license (http://creativecommons.org/licenses/by/4.0/). 